fifty-two reflexions, among the 890 observed by Carlisle, Scouloudi and Spier (private communication) for ribonuclease $(h 0 l)$, in four constellations, was carried out, using Beevers-Lipson strips without any calculating machines, in five days. The result is shown in the accompanying diagram. It shows a number of peaks of varying height which seem to be at intervals averaging $5 \mathrm{~A}$. in somewhat wavy alignments also $5 \mathrm{~A}$. apart in the direction of the c-axis, which may correspond to the direction of protein chains. While not claiming that this presents a true picture of the protein molecule, it can be claimed that it brings out some of its invariant character, namely, the part dependent only on the intensities and not the phases of the X-ray diffraction pattern. Fuller results, including a general theory of phase determination, will be published elsewhere.

Department of Physics,

$$
\text { J. D. Bernat }
$$

Birkbeck College,

University of London, London, W.C.1. April 29.

1 Harker, D., and Kasper, J. S., Acta Cryst., 1, 70 (1948

\section{Crystal Structures of Two Para-substituted Phenylpropionic Acids}

B-PHENYLPROPIONIC acid is of particular interest as an inhibitor in the carboxypeptidase system ${ }^{1}$, in addition to its general interest in biology. One of $u^{2}$ has shown that the unsubstituted acid is not amenable to $\mathrm{X}$-ray analysis, having eight molecules in a monoclinic primitive cell. We have therefore studied the para-substituted chlorine and bromine compounds. They are isomorphous, with cell data as follows :

$\beta$-(p-chlorphenyl)-propionic acid : $a=30.01$ A., $b=5.078$ A., $c=5 \cdot 744$ A., $\beta=98^{\circ} 30^{\prime}$, $P 2_{2} / a$;

$\beta$ - $(p$-bromphenyl $)$-propionic acid :

$a=30 \cdot 15$ A., $b=5 \cdot 143$ A., $c=5 \cdot 827$ A., $\beta=98^{\circ} 40^{\prime}$, $P 2_{1} / a$,

in each case with four molecules per unit cell. Weissenberg multiple-film techniques were used in visual evaluation of the reflexion intensities, and the precession camera was used in determining the cell dimensions and in checking the orientation adopted. The [010] projections are well resolved for both these substances and are quite similar. That for the chlorine compound is illustrated in the accompanying diagram. The [001] projections are less well resolved.

Preliminary analysis of the data for the chlorine compound indicates that the chlorine atom, the benzene ring, and the $\alpha$ - and $\beta$-carbon atoms of the aliphatic acid chain lie very close to one plane, not

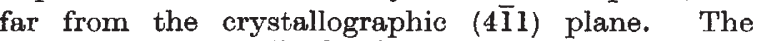
carboxyl group is tilted a few degrees from the plane of the rest of the molecule and is linked by hydrogen bonds to the carboxyl group of a second molecule related to the first by a symmetry centre. The bondlength of the $\alpha-\beta$ bond is close to the single-bond value, whereas the other two $\mathrm{C}-\mathrm{C}$ bonds in the chain appear shortened.

Details of bond-lengths and angles will be discussed elsewhere after further refinement has been carried

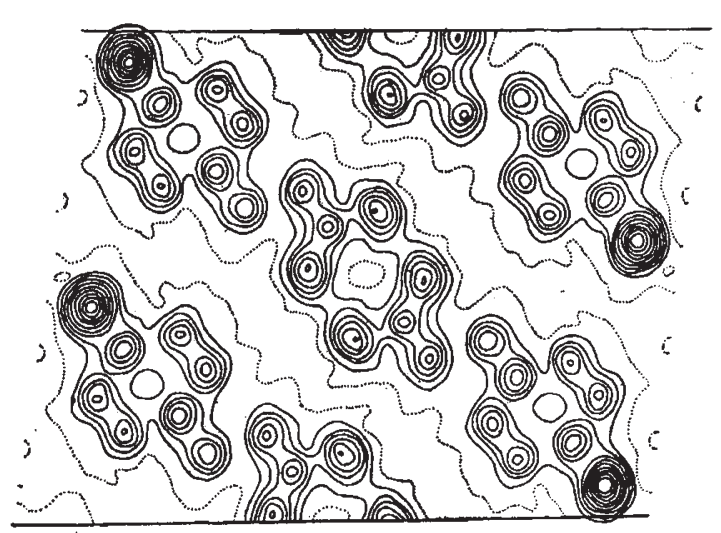

Plot of the [010] projection of the electron density of $\beta$ - $(p$-chlorphenyl)-propionic acid. The contour interval is 1 e/A. ${ }^{2}$ except within the chlorine atom above $4 \mathrm{e} / \mathrm{A} .{ }^{2}$, where the interval is $2 \mathrm{e} / \mathrm{A} .{ }^{2}$. The $1 \mathrm{e} / \mathrm{A} .^{2}$ contour is dotted. The portion plotted has a horizontal edge $a / 2$ and an oblique edge $2 c$. The origin is in a centre of symmetry projected at the centre of the plot

out. Studies are also in progress on the related trans- $\beta$-phenylacrylic acids, and on the orthosubstituted phenylpropionic acids, which are of interest in enzyme studies.

We are indebted to the National Cancer Institute of the National Institutes of Health, U.S. Public Health Service, for grants in support of this research, to Prof. Ernst Berliner, of Bryn Mawr College, who supplied the two compounds investigated, and to Mrs. Alice Sword Weldon for assistance with computations.

Institute for Cancer Research and

A. L. Patterson

JoAN R. CLARK

Lankenau Hospital Research Institute,

Philadelphia 11, Pennsylvania. April 1.

${ }^{1}$ For example, see Smith, Emil L., and Lumry, R., Cold Spring Harbor Symp. Quant. Biol., 14, 168 (1950).

2 Patterson, A. L., Phil. Mag., 3, 1252 (1927).

\section{Measurement of the Thickness of Transparent Films with the Light-Profile Microscope}

THE light-profile microscope technique, described by Tolansky ${ }^{1}$, has been adapted by us for the determination of the optical thickness of relatively thick transparent films. A transparent film, bounded by media with refractive indices differing from that of the film, has two reflecting surfaces; when observed with a light-profile microscope these two surfaces produce a double image, from the separation of which it is relatively easy to derive the ratio $t / \mu$, in which $t$ is the thickness and $\mu$ the refractive index. ' $\mathrm{t}$ 'his is of much value in so far as direct interferometry, using, for example, fringes of equal chromatic order, gives the value $\mu t$. The combined methods thus give both $\mu$ and $t$.

For films exceeding a few microns in thickness, difficulties due to depth of focus prevent precise focus simultaneously on both images. We have found it satisfactory to set at an intermediate focal plane, such that both images are defocused by about the same amount. That the compromise is satisfactory is shown by a thickness evaluation of 23 microns with an 8-mm. objective (depth of focus 2 microns) for a sheet of mica of thickness known to be $22 \frac{1}{2}$ microns. 